\title{
Estudio Morfológico del Cerebro y Pares Craneales de Diplobatis ommata (Elasmobranchii: Narcinidae)
}

\author{
Morphological Study of the Brain and Cranial Nerves \\ of Diplobatis ommata (Elasmobranchii: Narcinidae)
}

\author{
Héctor Marcos Montes Domínguez; Raquel López Bárcenas* \& Mónica González Isáis*
}

MONTES, D. H. M.; LÓPEZ, B. R. \& GONZÁLEZ, I. M. Estudio morfológico del cerebro y pares craneales de Diplobatis ommata (Elasmobranchii: Narcinidae). Int. J. Morphol., 32(4):1152-1155, 2014.

RESUMEN: El grupo de los Torpediniformes representa una de las líneas ancestrales de los batoideos cuya ruta evolutiva ha seguido una línea separada del resto de este grupo. No se han realizado estudios sobre la morfología del encéfalo de Diplobatis ommata, es por ello que el objetivo de este estudio fue describir la anatomía del encéfalo y pares craneales de esta especie. Los organismos fueron colectados en Zihuatanejo, Guerrero, México. El encéfalo se obtuvo por medio de la técnica de disección convencional. El encéfalo de Diplobatis ommata es similar al de los tiburones basales, sin embargo, presenta una vesícula eléctrica, característica de Torpediniformes. Las estructuras más grandes del encéfalo son los bulbos olfatorios y la médula oblongada. El telencéfalo es reducido y el cerebelo presenta simetría bilateral y es prácticamente liso. La disposición de los pares craneales es similar a la que presentan otros grupos de batoideos, sin embargo, la inervación de los pares craneales VII, IX y X se modifica, ya que además de inervar a las regiones descritas convencionalmente, inervan también a los órganos eléctricos.

PALABRAS CLAVE: Elasmobranchii; Torpediniformes; Diplobatis; Cerebro; Pares craneales.

\section{INTRODUCCIÒN}

Las rayas eléctricas (Torpediniformes) son elasmobranquios vivíparos aplacentados que se encuentran en la mayoría de los océanos (Neer \& Calliet, 2001) y representan aproximadamente el $8 \%$ del total de los batoideos (Compagno, 1990). Se caracterizan por presentar aletas pectorales redondeadas, cola larga y fuerte y boca protráctil; este grupo posee órganos eléctricos a cada lado de la cabeza con los que generan descargas eléctricas para su defensa.

El estilo de vida de cualquier organismo se refleja en la organización del sistema nervioso central, por lo que el estudio del encéfalo puede proporcionar información valiosa sobre la biología de cualquier organismo. Los cerebros grandes se correlacionan con factores como la complejidad del hábitat, la función locomotora y un estilo de vida como depredador activo. El tamaño relativo de las áreas sensoriales del cerebro, que reflejan especializaciones y la importancia de una modalidad sensorial dada, tienden a estar estrechamente relacionados con la alimentación. El desarrollo de las áreas de integración, como el telencéfalo y el cerebelo, han sido relacionadas con las diferencias en el microhábitat (Lisney \& Collin, 2006).
Los trabajos que se han realizado con el encéfalo de elasmobranquios se han enfocado al estudio de la relación masa del encéfalo - masa del cuerpo (Northcutt, 1978; Yopak \& Montgomery, 2008; Lisney et al., 2008), en los cuales se ha encontrado que el encéfalo de algunos grupos de batoideos es similar al tamaño de algunas aves y mamíferos. Autores como Lisney et al., Yopak \& Montgomery y Yopak \& Frank (2009), se han dedicado a estudiar la organización y el tamaño del cerebro considerando las relaciones filogenéticas y la complejidad del hábitat. Los trabajos que se han realizado sobre la morfología externa del encéfalo son pocos, destacando el trabajo de Walker \& Sherman (2001) los cuales describen de manera detallada la anatomía externa del encéfalo de Urobatis jamaicensis. Sin embargo, la mayoría de los estudios abordan de manera general a los grupos principales (Smeets, 1998; Hofmann, 1999) o de manera particular alguna estructura del encéfalo (Puzdrowski \& Leonard, 1992; New, 2001). Es por ello que el objetivo de este trabajo fue realizar un estudio de anatomía de la morfología externa del encéfalo y pares craneales de Diplobatis ommata. 


\section{MATERIAL Y MÉTODO}

Se utilizaron 10 organismos de Diplobatis ommata, los cuales fueron recolectados en las costas Ixtapa Zihutanejo, Guerrero, México. De cada organismo el cerebro fue expuesto y fijado con formol salino al $4 \%$.

En el laboratorio los cerebros fueron disectados mediante la técnica convencional para observar el encéfalo y los pares craneales. Se tomaron longitud y anchura máxima del telencéfalo, diencéfalo, mesencéfalo, cerebelo y médula oblongada. Las medidas fueron expresadas como porcentaje de la longitud patrón, la cual fue considerada desde la parte cefálica del telencéfalo hasta la parte caudal de la médula oblongada. La terminología utilizada se basó en la empleada por Northcutt.

\section{RESULTADOS}

Diplobatis ommata tiene un cerebro alargado en el que se distinguen de manera clara las regiones principales. En la parte anterior, en vista dorsal (Fig. 1), se encuentra el telencéfalo; es un estructura lisa, redondeada, que está dividida longitudinalmente por medio de un surco poco profundo, su longitud promedio es de $27,6 \%$ de la longitud patrón. En la región anterolateral del telencéfalo se encuentran los lóbulos olfatorios. A partir de los cuales se originan los tractos olfatorios, que son muy largos y delgados, estos llegan a la región media de los bulbos olfatorios, que se encuentran dentro de las cápsulas nasales. Los bulbos son grandes y ocupan en promedio el $27 \%$ de la longitud patrón.

El diencéfalo es simétrico, su longitud promedio es de aproximadamente $17,29 \%$ de la longitud patrón. Se encuentra en posición caudal al par craneal II (Fig. 2). Posterior a este se encuentran los lóbulos inferiores del infundíbulo, divididos por el lóbulo rostral de la hipófisis.

El mesencéfalo es liso, presenta simetría bilateral, se ubica en posición caudal al telencéfalo (Fig. 1). Su longitud aproximada es un $20,02 \%$ de la longitud patrón.

El cerebelo es simétrico, tiene una forma romboide y no presenta foliaciones. Está dividido en cuatro vesículas bien diferenciadas por una fisura transversal y una longitudinal. Se pueden observar otros 4 surcos poco profundos, dos longitudinales y dos en los extremos en forma de "V". Esta estructura ocupa en promedio el 23,79\% de la longitud patrón. En posición caudal al cerebelo se encuentra una vesícula eléctrica pequeña (Fig. 1).

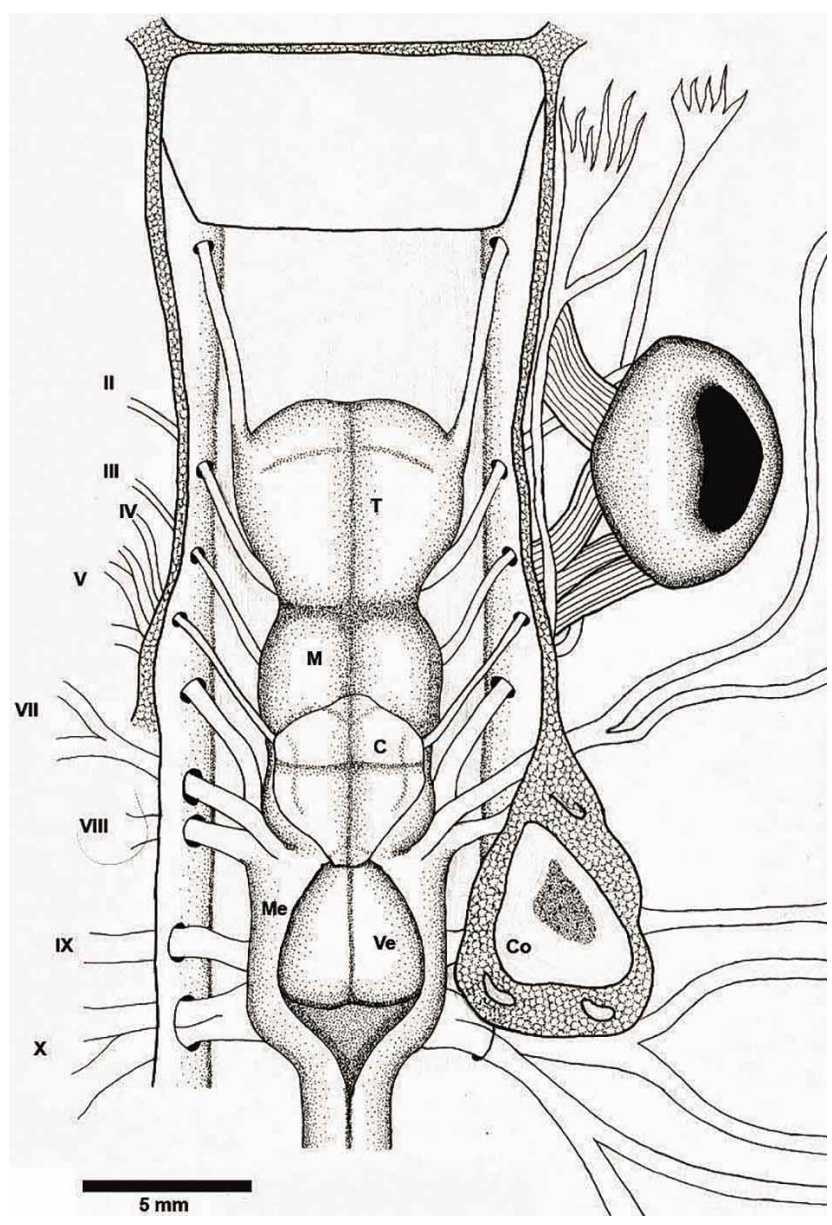

Fig. 1. Esquema de la vista dorsal del encéfalo de Diplobatis ommata $. \mathrm{C}=$ cerebelo; $\mathrm{Co}=$ cápsula ótica; $\mathrm{M}=$ mesencéfalo; $\mathrm{Me}=$ médula oblongada; $\mathrm{T}=$ telencéfalo; $\mathrm{Ve}=$ vesícula eléctrica; $\mathrm{I}-\mathrm{X}=$ pares craneales.

La médula oblongada es una estructura alargada, lisa y se ubica posterior al diencéfalo (Fig. 2) por debajo del cerebelo y de la vesícula eléctrica, se observa claramente la fosa romboidea posterior a las vesículas eléctricas en la parte caudalde la médula oblongada. Esta es la estructura más larga del encéfalo y ocupa en promedio el $47,9 \%$ de la longitud patrón.

El origen e inserción de los pares craneales es similar a lo reportado para otros elasmobranquios, sin embargo, existen algunas diferencias, las cuales se deben principalmente a las presencia de los órganos eléctricos. Se observó que los pares craneales facial (VII), glosofaríngeo (IX) y el vago (X) inervan a los órganos eléctricos (Fig. 3), por lo que se describen detalladamente. El nervio facial se origina en la médula oblongada detrás de la aurícula del cerebelo (Fig. 1), sin embargo, una parte de este nervio también tiene su origen en la región posterior de la aurícula, está dividido en dos ramas principales, la primera es más ancha y se dirige anteriormen- 


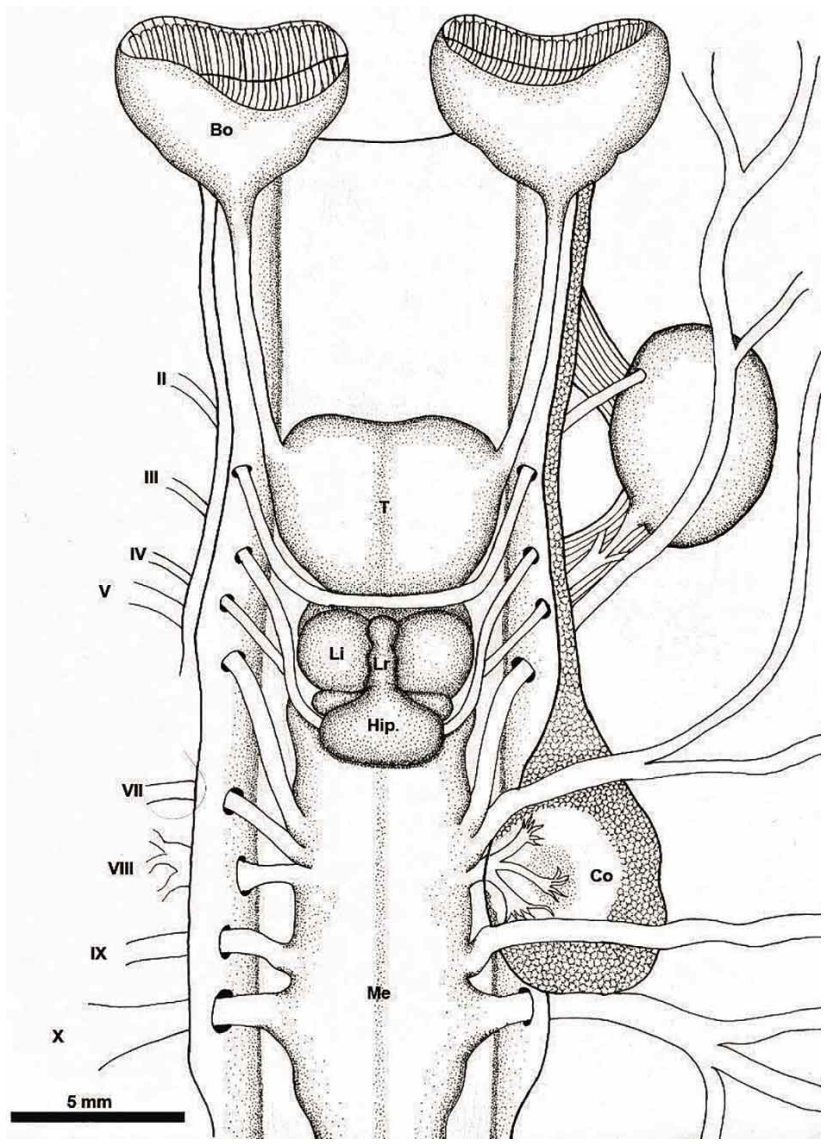

Fig. 2. Esquema de la vista ventral del encéfalo de Diplobatis ommata . $\mathrm{Bo}=$ bulbos olfatorios; $\mathrm{Co}=$ cápsula ótica; Hip= hipófisis; $\mathrm{Li}=$ lóbulos inferiores del infundíbulo; $\mathrm{Lr}=$ lóbulo rostral de la hipófisis; $\mathrm{Me}=$ médula oblongada; $\mathrm{T}=$ telencéfalo; $\mathrm{I}-\mathrm{X}=$ pares craneales.

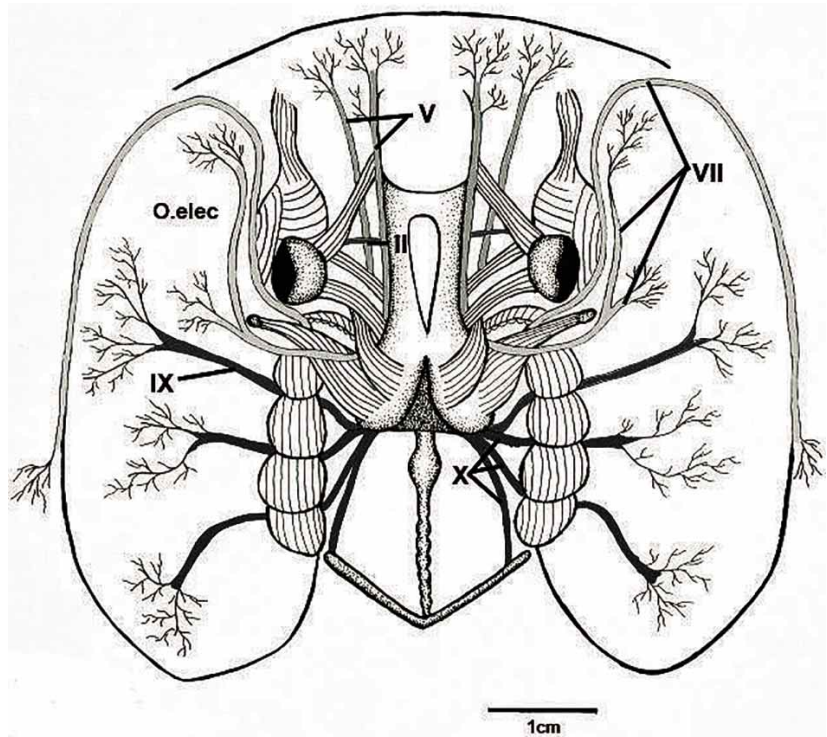

Fig. 3. Vista dorsal del encéfalo de Diplobatis ommata, en donde se observa como los pares craneales VII, IX y X inervan a los órganos eléctricos. $\mathrm{O}$. elec $=$ órganos eléctricos. te, inervando la región anterior de la cabeza y al músculo preorbital lateral en la cara lateral interna del músculo. La segunda rama se origina de la aurícula del cerebelo pasando por debajo de la primera rama, se dirige lateralmente y se subdivide una parte inerva todo el borde del disco hasta la parte media de los órganos eléctricos, y la segunda subdivisión inerva la región anterior del órgano eléctrico (Figs. 1 y 3).

El glosofaríngeo se origina en la médula oblongada (Fig. 1) y se dirige lateralmente pasando por el foramen del nervio glosofaríngeo, pasa por la región branquial inervándola y se inserta en la región media del órgano eléctrico en donde se ramifica (Fig. 3).

El vago se origina en la región caudal de la médula oblongada detrás del par craneal IX (Fig. 1) . Tiene una clara división en donde se observan dos ramas gruesas. Se dirige lateralmente pasando por el foramen del nervio vago, una de las ramas inerva la región branquial y se dirige al órgano eléctrico en donde se ramifica, la segunda rama se divide en donde una de las ramas (la más ancha) se dirige lateralmente inervando la región branquial y la parte posterior del órgano eléctrico (Fig. 3), la siguiente rama se dirige caudalmente inervando la región visceral del organismo.

\section{DISCUSIÓN}

En Diplobatis ommata, se observa una de las estructuras más grandes son los bulbos olfatorios, en seguida sigue la médula oblongada (47,9\% de la longitud patrón) y el telencéfalo $(27,59 \%)$. El diencéfalo es la estructura más pequeña $(17,29 \%)$. El alto grado de desarrollo de los bulbos olfatorios, nos indica que el sentido del olfato es muy importante en este grupo, lo cual es coherente dado que los ojos de estos organismos están reducidos.

El telencéfalo de D. ommata está moderadamente desarrollado, lo cual según Smeets sugiere una conducta social relativamente moderada. El cerebelo presenta simetría bilateral y es liso, similar al reportado para quimeras y squalomorfos, lo que sugiere una condición ancestral.

Diplobatis ommata presenta una vesícula adicional en el encéfalo, conocida como vesícula eléctrica. Esta estructura se ha reportado para Torpedo nobiliana (Smeets), sin embargo, en esta última especie la vesícula eléctrica está tan desarrollada, que es la estructura de mayor tamaño del encéfalo.

Con respecto a los pares craneales, en D. ommata se puede mencionar que la mayoría concuerda con las descripciones de los diferentes autores para elasmobranquios, sin em- 
bargo, los pares craneales V, VII y VIII, no tienen un origen común como se ha reportado en Squalus acanthias (Walker \& Sherman; da Silva Casas et al., 2005). En U. jamaicensis está separado el par V, sin embargo los pares VII y VIII, comparten un origen común (Walker \& Sherman). da Silva Casas $e t$ al. mencionan que en Carcharias taurus el origen de los pares V, VII y VIII no es el mismo, condición que también presentan algunos miembros de la familia Squalidae y algunos rajoideos, esto concuerda con lo encontrado en $D$. ommata, ya que en las descripciones dadas para $C$. taurus se menciona que en vista ventral, el origen aparente del par VII se localiza caudalmente al par $\mathrm{V}$, condición muy similar encontrada en D. ommata. Sin embargo, en D. ommata se presenta una condición particular, a diferencia de otras especies de batoideos, los pares craneales VII, IX y X, además de inervar las estructuras descritas en otras especies, inervan también a los órga- nos eléctricos, es posible que esta condición se presente en la mayoría de las especies de Torpediniformes, sin embargo aún no se ha descrito.

Finalmente, es posible concluir que el encéfalo de Diplobatis ommata presenta un telencéfalo relativamente pequeño y una médula oblongada alargada y bien desarrollada, similar a lo reportado para tiburones basales (squalomorfos) y holocéfalos (Hoffman). Se ha mencionado que los Torpediniformes representan una de las líneas ancestrales de los batoideos cuya ruta evolutiva ha seguido una línea separada del resto de este grupo. Esto se ve reflejado en la morfología del encéfalo, ya que en él se observa la presencia de una vesícula eléctrica característica del grupo, además la inervación de los pares craneales se modifica, debido a la presencia de los órganos eléctricos.

MONTES, D. H. M.; LÓPEZ, B. R. \& GONZÁLEZ, I. M. Morphological study of the brain and cranial nerves of Diplobatis ommata (Elasmobranchii: Narcinidae). Int. J. Morphol., 32(4):1152-1155, 2014.

SUMMARY: The Torpediniformes group is one of the ancestral batoid lines, the evolution of which has followed a line separated from the rest of this group. No studies have been conducted on the brain morphology of Diplobatis ommata. This is why the objective of this work is describing the anatomy of the brain and the cranial nerves of this species. Organisms were collected in Zihuatanejo, Guerrero, Mexico. The brain was obtained through conventional dissection techniques. The brain of Diplobatis ommata is similar to that of basal sharks; however, it has the electric vesicle typical in Torpediniformes. The largest encephalic structures are the olfactory bulbs and medulla oblongata. The telencephalon is reduced, while the cerebellum has bilateral symmetry and is almost smooth. Disposition of cranial nerves are similar to other batoid groups; but innervation of pairs VII, IX and X is modified: they innervate regions conventionally described, but also electric organs.

KEY WORDS: Elasmobranchii; Torpediniformes; Diplobatis; Brain; Cranial nerves.

\section{REFERENCIAS BIBLIOGRÁFICAS}

Compagno, L. J. V. Alternative life-history styles of cartilaginous fishes in time and space. Eviron. Biol. Fish., 28(1-4):33-75, 1990.

da Silva Casas, A. L. da; Intelizano, W.; Fernandes de Souza Castro, M. \& Bonfim Mariana, A. N. Nerves of the Mandibular Musculature of the Sand Tiger Shark Carcharias taurus (Rafinesque, 1810) (Chondrichthyes: Odontaspididae). Int. J. Morphol., 23(4) :387-92, 2005.

Hoffman, M. H. Nervous system. En: Hamlet, W. C. (Ed.). Sharks, Skates, and Rays: The Biology of Elasmobranch Fishes. Baltimore, Johns Hopkins University Press, 1999.

Lisney, T. J. \& Collin, S. P. Brain morphology in large pelagic fishes: a comparison between sharks and teleosts. J. Fish Biol., 68(2):532-54, 2006.

Lisney, T. J.; Yopak, K. E.; Montgomery, J. C. \& Collin, S. P. Variation in brain organization and cerebellar foliation in chondrichthyans: batoids. Brain Behav. Evol., 72(4) :262-82, 2008.

Neer, J. A. \& Calliet, G. M. Aspects of the life history of the Pacific electric ray, Torpedo californica (Ayers). Copeia, 2001(3):842-47, 2001.

New, G. J. Comparative neurobiology of the elasmobranch cerebellum: theme and variations on a sensorimotor interface. Environ. Biol. Fish, 60(13):93-108, 2001.

Northcutt, R. G. Brain organization in the cartilaginous fishes. En: Hodgson, E. S. \& Mathewson, R. F. (Eds.). Sensory Biology of Sharks, Skates, and Rays. Arlington, Office of Naval Research, 1978.
Puzdrowski, R. L. \& Leonard, R, B.Variations in cerebellar morphology of the Atlantic stingray, Dasyatis sabina. Neurosci. Lett., 135(2):196-200, 1992.

Smeets, W. J. A. J. Cartilaginous fishes. En: Nieuwenhuys, R. \& Roberts, B. L. (Eds.). The Central Nervous System of Vertebrates, Berlin, SpringerVerlag, 1998.

Walker, B. K. \& Sherman, R. L. Gross brain morphology in the yellow stingray, Urobatis jamaicensis. Florida Sci., 64(4):246-9, 2001.

Yopak, K. E. \& Montgomery, J. C. Brain organization and specialization in deep-sea chondrichthyans. Brain Behav. Evol., 71(4):287-304, 2008.

Yopak, K. E. \& Frank, L. R. Brain size and brain organization of the whale shark, Rhincodon typus, using magnetic resonance imaging. Brain Behav. Evol., 74(2):121-42, 2009.

Dirección para Correspondencia:

Dra. Mónica González Isáis

FES Iztacala, UNAM

Lab. de Anatomía Animal Comparada

FES Iztacala, UNAM

Los Reyes Iztacala, Tlalnepantla

Estado de México, C.P. 54090

MÉXICO

Email: monigi@unam.mx

Recibido : 21-01-2014

Aceptado: 21-08-2014 